\title{
Glans reconstruction with the use of an inverted urethral flap after distal penile amputation for carcinoma
}

\author{
Salvatore Sansalone ${ }^{1}$, Giulio Garaffa ${ }^{2}$, Giuseppe Vespasiani ${ }^{1}$, Alessandro Zucchi ${ }^{3}$, \\ Franklin Emmanuel Kuehhas ${ }^{4}$, Ralf Herwig ${ }^{4}$, Mauro Silvani ${ }^{5}$, Stefano Pecoraro ${ }^{6}$, \\ Carla Loreto $^{7}$, Rosario Leonardi ${ }^{8}$ \\ ${ }^{1}$ Department of Experimental Medicine and Surgery, Tor Vergata University of Rome, Rome, Italy; \\ ${ }^{2}$ St Peter's Andrology, University College London Hospitals, London, UK; \\ ${ }^{3}$ Department of Urology and Andrology, University of Perugia, Perugia, Italy; \\ ${ }^{4}$ Department of Urology, Medical University of Vienna, Vienna, Austria; \\ ${ }^{5}$ Department of Urology, General Hospital, Biella, Italy; \\ ${ }^{6}$ Department of Nephro-Urology, Malzoni Medical Center Avellino, Avellino, Italy; \\ ${ }^{7}$ Department of Bio-Medical Sciences, Anatomy Section, University of Catania, Catania, Italy; \\ ${ }^{8}$ Musumeci GECAS Clinic of Catania, Catania, Italy.
}

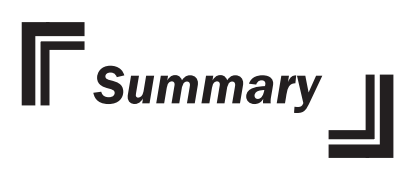

Restoration of adequate cosmesis and preservation of sexual and urinary function are the main goals of penile reconstructive surgery following amputation for carcinoma.

Split thickness skin grafts and oral mucosa grafts have been widely used for the creation of a pseudoglans with excellent cosmetic and functional results. The main drawbacks associated with the use of grafts are donor site morbidity, the lack of engorgement of the pseudoglans and the risk of poor graft take, which may lead to contracture and poor cosmetic results.

In the present series the long term cosmetic and functional outcomes of glans reconstruction with an inverted distal urethral flap are described.

KEY WORDS: Glans reconstruction; Urethral flap; Penile cancer.

\section{INTRODUCTION}

Squamous cell carcinoma of the penis (SCC), which represents a rare condition in Western Europe and North America, accounting for less than 1\% of all male malignancies, is slightly more common in the developing countries, where it has an incidence up to 20 per 100,000 people $(1,2)$.

The traditional treatment of SCC has been radical penectomy, which guarantees excellent local control rates at the expense of a complete loss of sexual and urinary function with consequent severe psychological morbidity (3).

Although there is little dispute that radical surgery is necessary for T3 and T4 disease, the need to perform this type of surgery in patients with T1 and T2 disease limited to the glans penis and the prepuce and the belief that a $2 \mathrm{~cm}$ margin is required to guarantee an adequate oncological clearance have been questioned in recent studies.
In particular, a more conservative organ sparing approach is suggested by the fact that more than $80 \%$ of SCC arises from the glans and prepuce and that margins of few millimeters may be adequate for the clearance of most tumours. Moreover, the penis is easy to inspect and therefore local recurrences can be immediately identified and treated (4-8).

Current EAU guidelines recommend a penile preserving approach for Carcinoma in situ, Ta, T1 and T2 SCC with involvement of the glans only in patients committed to a regular surveillance program (2).

In particular, patients with $\mathrm{T} 1$ and $\mathrm{T} 2$ disease limited to the glans penis should be offered glansectomy with or without corporeal tips amputation followed by construction of a pseudoglans $(9,10)$.

Herein the long-term outcome of a series of patients who 
have undergone organ-sparing surgery for the management of T1 and T2 SCC of the glans penis followed by reconstruction of a pseudoglans with the use of urethral flaps is reported.

\section{Materials AND Methods}

In our Institution, between March 2007 and May 2011, 34 patients have undergone organ-sparing surgery for the management of $\mathrm{T} 1$ and T2 SCC of the glans penis followed by pseudoglans reconstruction with the use of an inverted distal urethral flap. All patients gave their informed consent prior to their inclusion in the study. Preoperatively, 22 patients reported good quality erection and 19 reported to be sexually active.

Prior to surgery all patients have undergone local stadiation based on clinical examination and on Magnetic Resonance Imaging (MRI) findings. In particular, contrast enhanced T1 and T2 weighted MRI images in combination with an artificial erection with prostaglandin E1 (PGE1) were used to identify invasion of the tumour through the tunica albuginea into the corpora cavernosa. Only sexually active patients committed to a regular surveillance program with clinical T1 and T2 disease arising from the glans and without involvement of the corpora cavernosa were included in the series.

Patients with a history of urethral stricture or of previous urethral surgery were excluded from this series and underwent glans reconstruction with split thickness skin grafts as previously described by various Authors (5, 8, 10-12). This because glans reconstruction with the use of urethral flap relies on an adequate blood supply form the proximal aspect of the urethra, and this might be severely compromised in patients with spongiofibrosis. After a circumferential subcoronal incision has been carried out in the shaft skin and deepened down to the level of Buck's fascia, the glans was dissected off the corporeal

Figure 1.

A circumferential subcoronal incision is carried out and the penile disassembly is performed.

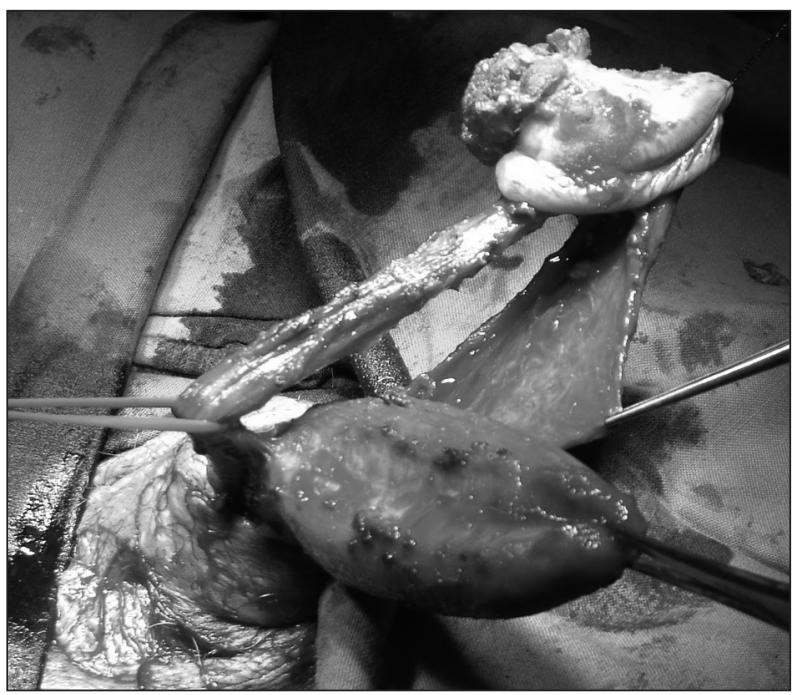

Figure 2.

The urethra is divided and then spatulated ventrally.

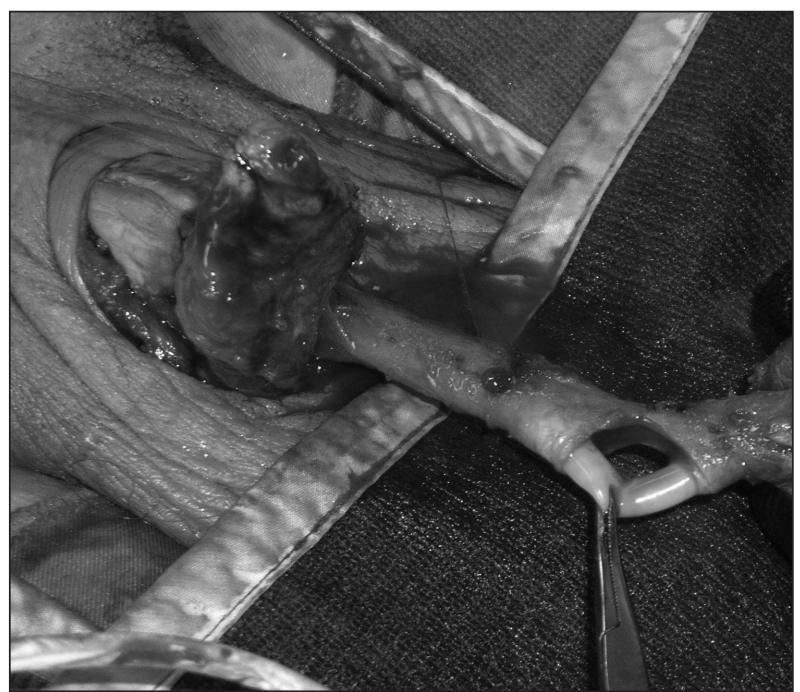

heads and the distal aspect of the urethra and frozen sections were collected form the corporeal heads and the distal urethral margin in all patients, as previously described by various Authors (5, 8, 10-15). Two patients presented positive corporeal margins at the frozen section and underwent distal corporectomy with reconstruction of the corporeal heads.

The shaft penis was then completely degloved in all patients in order to allow a complete dissection of the urethra off the corpora cavernosa down to the crura (Figure 1). The distal aspect of the urethra was then spatulated ventrally for approximately $2.5 \mathrm{~cm}$, everted and used to cover the corporeal heads in order to form a pseudoglans (Figure 2). The urethral edges were sutured to the underlying corpora cavernosa with interrupted 4-0 Polyglactin sutures (Vicryl ${ }^{\circledR}$, Ethicon, Somerville, New Jersey, USA) in order to form the ridge of the pseudoglans. The penile shaft skin was also approximated to the edge of the urethral flap using interrupted 4-0 Polyglactin sutures in order to recreate the coronal grove (Figure 3).

A light compressive dressing was applied on the penile shaft to prevent haematoma formation and a 16 French Foley catheter left in situ for 24 hours. Patients were usually discharged on postoperative day one on broad-spectrum oral antibiotics for 1 week.

Patients were reviewed on postoperative week 2 , after 3 months and then on a 6 monthly basis and instructed to regularly inspect the genitalia in order to identify early signs of recurrence. A physical examination was routinely performed at each visit while a Computerized Tomography (CT) scan of chest, abdomen and pelvis with contrast was carried out on a yearly basis. Patients with histology of pT1 G3 and pT2 and/or palpable groin disease have been then managed with groin node dissection according to the EAU guidelines (2).

Cosmetic and functional outcome of the glans reconstruction, patients' satisfaction, complications, recurrence of the disease and eventual need for revision surgery were recorded in the postoperative follow-up visit. 
Figure 3.

The final results after the eversion of the urethral flap and the creation of the pseudoglans

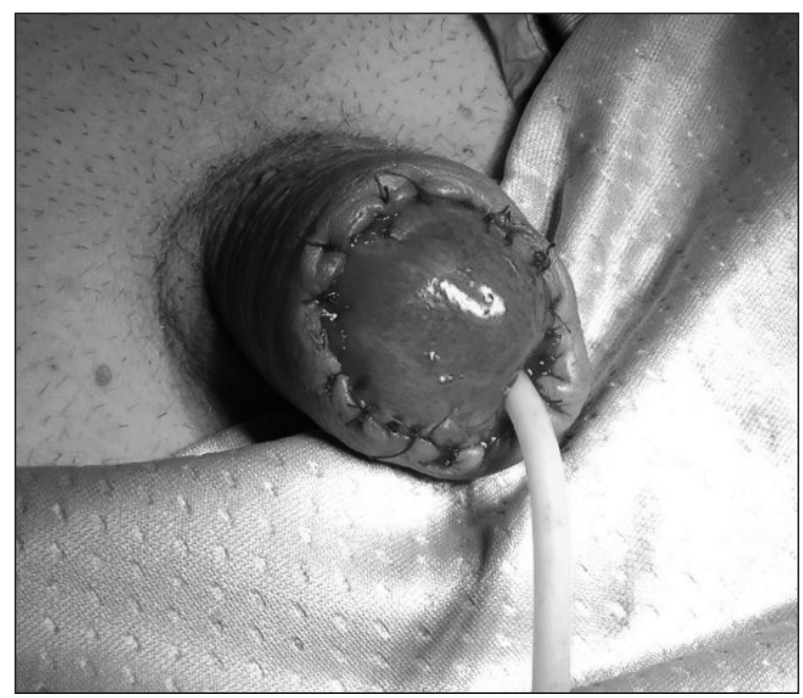

Sensation of the urethral flap and patient's satisfaction were assessed by direct questioning the patient and no objective test or validated questionnaire has been employed.

\section{ResUlts}

Mean age at the time of surgery was 60 years (range 3673) and the final histo-pathological results are reported in Table 1.

Infiltration of the proximal surgical margin, which had resulted clear in the initial frozen section, was present in 4 patients with pT2 disease (17\%) and was managed with a distal corporectomy followed by glans reconstruction with the use of split thickness skin grafts, as the distal urethra had already been used (5).

After a median follow-up of 27 months (range 6-48 months) none of the patients has succumbed because of the disease.

The urethral flap survived in all patients and a penile hematoma occurred in 2 (6\%) patients and required surgical evacuation in one case.

A local recurrence occurred in one of the patients who had pT2 disease (3\%) and was noticed at the 6 months follow up visit. Eventually the patient was managed with partial penectomy and was local recurrence free at the 18 months follow up visit.

Table 1.

Histology results.

\begin{tabular}{|l|c|}
\hline Histology result & N. of patients \\
\hline G2 pT1 & 12 \\
\hline G3 pT1 & 15 \\
\hline pT2 & 7 \\
\hline
\end{tabular}

All patients who did not require further local surgery were able to maintain physiological urinary function and considered the cosmetic result of the urethral flap satisfactory. All 22 patients who preoperatively had good quality erections reported no reduction in rigidity after surgery. Overall, 14 of the patients who were preoperatively sexually active have resumed sexual activity postoperatively (74\%). Of the remainder, 4 had required a delayed partial penectomy due to malignant infiltration of the corporeal heads and one, who had undergone glansectomy followed by bilateral radical lymph node dissection of the groin, had a residual penile length insufficient for penetration. It is likely that the prepubic and scrotal lymphoedema consequence of the bilateral lymph node dissection was the cause of the perceived loss of penile length in this patient.

A ventral penile curvature during erection was noticed by 2 patients (9\%) and was consequence of a relatively short urethra, which was acting as a ventral chordee. This occurred despite of a meticulous intraoperative dissection of the urethra proximally down to its bulbar aspect. In both cases the curvature was less than 20 degrees, and, although ventral, did not interfere significantly with sexual activity.

At 6 months postoperative follow up, all patients who have not required further local surgery have reported pseudo glans sensation and a degree of urethral flap engorgement.

\section{Discussion}

Reconstruction of the glans penis following glansectomy or distal corporectomy for carcinoma of the penis represents a challenge for the reconstructive surgeon. This because the aim of surgery is to achieve complete oncological clearance and to guarantee adequate cosmetic and functional results to allow the patient to resume sexual and urinary function with confidence (5-15).

After the initial technique description in 2004, split thickness skin grafts, usually harvested from the inner thigh, have been widely used for glans resurfacing and pseudoglans construction for both benign and malignant conditions (5, 8-18). A recent series has also described the use of oral mucosa grafts for glans reconstruction.18 Although glans reconstruction with the use of grafts yields adequate cosmetic and functional results in the hands of experienced surgeons, it is associated with donor site morbidity and the risk of poor graft take, which can lead to contracture and poor cosmesis. Despite a good cosmetic result, the pseudoglans fashioned with this technique does not engorge like the native one (8). Furthermore, oral infection with human papillomavirus (HPV), which can have a prevalence as high as 31\%, can lead to the development of HPV related carcinomas arising from the oral mucosa grafts, although the literature has not provided any indication of the actual risk after genital reconstruction (20).

This series confirms that a distal urethral flap is a good alternative to a split thickness skin graft for the reconstruction of the glans penis following amputation for carcinoma, as previously described in 1 series of 14 patients (21). This technique is not associated with donor site 
morbidity, as it does not require the harvesting of a graft, and as the spongy tissue engorges during the erection, it leads to tumescence of the pseudo glans and therefore produces a more physiological outcome.

Regardless to the reconstructive technique used, glansectomy and distal corporectomy needs to be carried out as previously described in the literature. 8 In particular, frozen sections of the corporeal and urethral margins are required to assess whether a complete clearance of the malignancy has been achieved $(8,22)$.

Only patients with a good blood supply to the distal urethra can be offered glans reconstruction with the use of distal urethral flap and therefore in presence of history of urethral strictures and/or previous urethral surgery reconstruction should be performed with the use of skin grafts. Patients need to be warned that even with adequate preparation of the urethra, which should be dissected proximally down to its bulbar portion, a minor ventral penile curvature during erection is not an uncommon finding.

\section{Conclusions}

The present series confirms that urethral flaps represent an excellent alternative to split thickness skin grafts for pseudoglans reconstruction following glansectomy and partial corporectomy for penile carcinoma in carefully selected patients.

\section{REFERENCES}

1. Mistry T, Jones RW, Dannat E, Pet al. A 10-year retrospective audit of penile cancer management in the UK. BJU Int. 2007; 100:1277-81.

2. Pizzocaro G, Algaba F, Horenblas S, et al. EAU penile cancer guidelines 2009. Eur Urol. 2010; 57:1002-12.

3. Opjordsmoen S, Fossa SD. Quality of life in patients treated with for penile cancer. A Follow-up study. Br J Urol. 1994; 74:652-7.

4. Hegarty PK, Shabbir M, Hughes B, et al. Penile preserving surgery and surgical strategies to maximize penile form and function in penile cancer: recommendations from the United Kingdom experience. World J Urol. 2009; 27:179-87.

5. Pietrzak P, Corbishley C, Watkin N. Organ sparing surgery for invasive penile cancer: early follow-up data. BJU Int. 2004; 94:1253-7.

6. Hoffman MA, Renshaw AA, Loughlin KR. Squamous cell carcinoma of the penis and microscopic pathologic margins: How much margin is needed for local cure? Cancer. 1999; 85:1555-68.

7. Minhas S, Kayes O, Hegarty P, et al. What surgical resection margins are required to achieve oncological control in men with primary penile cancer? BJU Int. 2005; 96:1040-3.

8. Smith Y, Hadway P, Biedrzycki O, et al. Reconstructive surgery for invasive squamous carcinoma of the glans penis. Eur Urol. 2007; 52:1179-85.

9. Lont AP, Gallee MP, Meinhardt W, et al. Penis conserving treatment for T1 and T2 penile carcinoma: clinical implications of a local recurrence. J Urol. 2006; 176:575-80.

10. Palminteri E, Berdondini E, Lazzari M, Barbagli G. Resurfacing and reconstruction of the glans penis. Eur Urol. 2007; 52:893-900.

11. Barbagli G, Sansalone S, Djinovic R, et al. Current controversies in reconstructive surgery of the anterior urethra: a clinical overview. Int Braz J Urol. 2012; 38:307-16.
12. Bracka A. Grans resection and plastic repair. BJU Int. 2010; 105:136-44.

13. Ralph DJ, Garaffa G, Garcia MA. Reconstructive surgery of the penis. Curr Opin Urol. 2006, 16:396-400.

14. Garaffa G, Sansalone S, Ralph DJ. Penile reconstruction. Asian J Androl. 2013; 15:16-9.

15. Loreto C, Garaffa G, Djinovic R, et al. Penile disassembly: anatomical surgical steps. BJU Int. 2013; (in press).

16. Garaffa G, Shabbir M, Christopher AN, et al. The surgical management of lichen sclerosus of the glans penis: our experience and review of the literature. J Sex Med. 2011; 8:1246-53.

17. Shabbir M, Muneer A, Kalsi J, et al. Glans resurfacing for the treatment of carcinoma in situ of the glans: surgical technique and outcomes. Eur Urol. 2011; 59:142-7.

18. Morelli G, Pagni R, Mariani C, et al. Glansectomy with split thickness skin graft for the treatment of penile carcinoma. Int J Imp Res. 2009; 21:311-4.

19. Venkov G, Laaser MK. Reconstruction of tissue defects of the glans penis by transplantation of buccal mucosa. Aktuelle Urol. 2008; 39:219-24.

20. Barbagli G, Sansalone S, Lazzeri M. Oral mucosa and urethroplasty: it is time to change. Eur Urol 2012; 62:1071-3.

21. Gulino G, Sasso F, Falabella R, Bassi PF. Distal urethral reconstruction of the glans for penile carcinoma: results of a novel technique at 1 year follow up. J Urol. 2007; 178:941-4.

22. Algaba F, Arce Y, Lopez-Beltran A, et al. Intraoperative frozen section diagnosis in urological oncology. Review. Eur Urol. 2005; 47:129-36.

\section{Correspondence}

Salvatore Sansalone, MD (Corresponding Author) salvatore.sansalone@yahoo.it

Giuseppe Vespasiani, MD

Department of Experimental Medicine and Surgery

Tor Vergata University of Rome - 00133 Rome, Italy

Giulio Garaffa, MD

St Peter's Andrology - University College London Hospitals

W1G 6BJ London, UK

Alessandro Zucchi, MD

Department of Urology and Andrology

University of Perugia - 06123 Perugia, Italy

Franklin Emmanuel Kuehhas, MD

Ralf Herwig, MD

Department of Urology - Medical University of Vienna

1040 Vienna, Austria

Mauro Silvani, MD

Department of Urology - Biella General Hospital

13900 Biella 13900, Italy

Stefano Pecoraro, MD

Department of Nephro-Urology, Malzoni Medical Center Avellino, 83100 Avellino, Italy

Carla Loreto, MD

Department of Bio-Medical Sciences - Anatomy Section

University of Catania - 95100 Catania, Italy

Rosario Leonardi, MD

Musumeci GECAS Clinic of Catania - 95100 Catania. Italy 